\title{
CONSUMO E DIGESTIBILIDADE DE NUTRIENTES DA TORTA DE DENDÊ NA DIETA DE OVINOS
}

\section{NUTRIENTS INTAKE AND DIGESTIBILITY OF PALM KERNEL CAKE IN SHEEP DIET}

\author{
Maiana Visoná-Oliveira ${ }^{1}$ \\ Isabel Cristina Ferreira² \\ Gilberto de Lima Macedo Junior ${ }^{3}$ \\ Luciano Fernandes Sousa ${ }^{4}$ \\ Jhone Talisson Lira de Sousa ${ }^{5}$ \\ Rogério Pereira dos Santos ${ }^{6}$

\begin{abstract}
${ }^{1}$ Médica Veterinária, Mestre pela Universidade Federal de Uberlândia, Uberlândia, MG, Brasil
2Pesquisadora A, Embrapa Cerrados, Área de Produção Animal, Brasília, DF, Brasil.

3 Professor Adjunto da Universidade Federal de Uberlândia, Uberlândia, MG, Brasil gilbertomacedojr@gmail.com

${ }^{4}$ Professor Adjunto da Universidade Federal do Tocantins, Araguaína, TO, Brasil

${ }^{5}$ Graduando em Zootecnia da Universidade Federal do Tocantins, Araguaína, TO, Brasil

${ }^{6}$ Zootecnista, Mestre pela Universidade Federal do Tocantins, Araguaína, TO, Brasil
\end{abstract}

\section{Resumo}

Objetivou-se encontrar o nível ótimo de inclusão da torta de dendê na dieta de ovinos. Foram utilizados 18 carneiros, castrados, sem raça definida alimentados com níveis de inclusão da torta de dendê de $0 \%, 7,5 \%, 15 \%$ e 22,5\% na dieta total. Os animais foram mantidos em gaiolas metabólicas por 21 dias. Os dados foram submetidos a estudo de regressão com 5\% de significância para as equações e coeficientes de regressão. A inclusão da torta de dendê provocou redução nos consumos de MS, hemicelulose, FDN, PB, NDT, CHOT e MM ( $\mathrm{P}<0,01)$ e não houve diferenças significativas nos de celulose, FDA e CNF $(\mathrm{P}>0,05)$. Os consumos de EE e lignina foram crescentes $(\mathrm{P}<0,01)$. $\mathrm{O}$ teor de fibra das dietas aliado ao consumo de lignina pelos animais provocou redução nos coeficientes de digestibilidade da EB e de todos os nutrientes avaliados (MS, PB, celulose, hemicelulose, FDN; $\mathrm{P}<0,05)$. Houve redução na metabolizabilidade das dietas devido ao aumento da EB fecal $(\mathrm{P}<0,01)$. O balanço de nitrogênio foi positivo em todos os tratamentos. A inclusão da torta de dendê acima de 7,5\% na dieta de ovinos provoca redução no CMS e na digestibilidade de nutrientes com possível comprometimento do desempenho, especialmente, em animais de alta produção.

Palavras-chave: balanço de energia; balanço de nitrogênio; Elaeis guineenses; lignina; ruminantes.

\begin{abstract}
The aim of this study was to find the optimum inclusion level of palm kernel cake in sheep diet. We used 18 castrated rams, fed diets with inclusion levels of palm kernel cake of $0 \%, 7.5 \%, 15 \%$ and
\end{abstract}


$22.5 \%$ of the total diet. Animals were housed in metabolic cages for 21 days. Data were subjected to regression study with $5 \%$ significance for equations and regression coefficients. The inclusion of palm kernel cake caused reduction in DM, hemicellulose, NDF, CP, TDN, TC and MM (P<0.01) and no significant differences in cellulose, ADF and NFC (P>0.05). The intake of EE and lignin increased $(\mathrm{P}<0.01)$. The fiber content of the diets added to the consumption of lignin by the animals caused reduction in digestibility of GE and all analyzed nutrients (DM, CP, hemicellulose, NDF; $\mathrm{P}<0.05)$. There was reduction in metabolizability of the diets due to increased GE of feces ( $\mathrm{P}$ $<0.01)$. Nitrogen balance was positive in all treatments. The inclusion of palm kernel cake above $7.5 \%$ in sheep diet causes reduction in DMI and digestibility of nutrients with possible performance losses, especially in high-performance animals.

Keywords: Elaeis guineenses; energy balance; lignina; nitrogen balance; ruminants.

Recebido em:29 julho 2013

Aceito em: 05 janeiro 2015

\section{Introdução}

A torta de dendê é o produto resultante da polpa seca do dendê, após moagem e extração do seu óleo $^{(1)}$. A substituição de ingredientes clássicos da alimentação de ruminantes por este coproduto da indústria do biodiesel é alternativa para a redução de custos a fim de se obterem sistemas produtivos mais sustentáveis ${ }^{(2)}$.

Quanto à composição químico-bromatológica encontrada na literatura revisada, a torta de dendê apresentou teor de MS entre 88,11 e 97,7\%, proteína bruta (PB) entre 13,01 e 18,21\%, extrato etéreo (EE) entre 5,7 e 13,55\%, fibra em detergente neutro (FDN) entre 64,09 e 81,85\%, fibra em detergente ácido (FDA) entre 41,29 e 56,02\% e matéria mineral (MM) entre 3,01 e 7,82\% ${ }^{(3-6)}$, o que a torna um produto não padronizado ${ }^{(3)}$.

A torta de dendê possui disponibilidade ao longo do ano, baixo custo e rica composição em nutrientes. Esses fatores, aliados ao provável aumento na produção mundial de óleo de dendê, em decorrência de seu uso na produção de biodiesel, possibilitarão maior acessibilidade a esse alimento, o que o torna uma alternativa promissora $^{(6)}$.

Assim, objetivou-se determinar níveis de inclusão da torta de dendê em dietas para ovinos por meio da avaliação do consumo, coeficientes de digestibilidade dos nutrientes, balanço de nitrogênio e balanço de energia.

\section{Material e métodos}

Este experimento foi aprovado pela comissão de ética na utilização de animais $\left(\mathrm{n}^{\circ}\right.$ 061/12) da Universidade Federal do Tocantins e teve duração de 21 dias, sendo 16 dias destinados à adaptação dos animais à dieta à vontade para estabilizar o consumo e cinco para coleta de dados. Os animais foram alojados em gaiolas metabólicas individuais próprias para o ensaio de digestibilidade in vivo, providas de comedouro e bebedouro. Cada gaiola metabólica possuía, acoplado ao assoalho, um 
sistema de captação total de fezes e urina.

Após pesagem, identificação e tratamento com anti-helmíntico, 18 ovinos machos, castrados, sem raça definida, com peso vivo médio de $36,8 \mathrm{~kg}$ e idade superior a 12 meses, foram distribuídos, em delineamento inteiramente casualizado, em quatro tratamentos com diferentes níveis de inclusão da torta de dendê na dieta total: $0 \%(n=5), 7,5 \%(n=5), 15 \%(n=4)$ e 22,5\% $(n=4)$. O experimento foi delineado com 20 animais. Contudo, dois animais morreram na fase de adaptação devido ao ataque de predadores e não foi possível substituí-los.

As dietas foram formuladas, segundo o $\mathrm{NRC}^{(7)}$, para se apresentarem isoenergéticas $\mathrm{e}$ isonitrogenadas. Elas eram compostas por silagem de Brachiaria brizantha cv. Marandu e concentrado à base de milho, farelo de soja, suplemento mineral e torta de dendê em diferentes níveis de inclusão. A proporção dos ingredientes e a composição químico-bromatológica da torta de dendê e da silagem estão descritas a seguir (Tabela 1).

Tabela 1: Proporção dos ingredientes das dietas com diferentes níveis de inclusão de torta de dendê e composição químico-bromatológica da torta de dendê e da silagem de $B$. brizantha cv. Marandu utilizadas

\begin{tabular}{|c|c|c|c|c|}
\hline \multirow{2}{*}{ Componentes } & \multicolumn{4}{|c|}{ \% Torta de dendê } \\
\hline & $\mathbf{0}$ & 7,5 & 15 & 22,5 \\
\hline \multicolumn{5}{|c|}{ Composição percentual (\%) } \\
\hline Silagem de $B$. brizantha cv. Marandú & 60,04 & 56,05 & 52,06 & 48,06 \\
\hline Torta de dendê & 0,00 & 7,50 & 15,00 & 22,50 \\
\hline Milho & 21,80 & 20,24 & 18,67 & 17,11 \\
\hline Farelo de Soja & 16,15 & 14,21 & 12,26 & 10,31 \\
\hline Suplemento mineral & 2,00 & 2,00 & 2,00 & 2,00 \\
\hline Total & 99,99 & 100 & 99,99 & 99,98 \\
\hline \multicolumn{5}{|c|}{ Composições químico-bromatológicas da torta de dendê e da silagem } \\
\hline Componentes & \multicolumn{2}{|c|}{ Torta de dendê } & \multicolumn{2}{|c|}{ Silagem } \\
\hline Matéria seca (\%) & \multicolumn{2}{|c|}{94,48} & \multicolumn{2}{|c|}{48,19} \\
\hline Proteína Bruta (\%) & \multicolumn{2}{|c|}{16,01} & \multicolumn{2}{|c|}{4,99} \\
\hline Nutrientes digestíveis totais (\%) & \multicolumn{2}{|c|}{64,00} & \multicolumn{2}{|c|}{50,66} \\
\hline Fibra em detergente neutro (\%) & \multicolumn{2}{|c|}{63,53} & \multicolumn{2}{|c|}{79,75} \\
\hline Fibra em detergente ácido (\%) & \multicolumn{2}{|c|}{41,19} & \multicolumn{2}{|c|}{47,73} \\
\hline Extrato etéreo (\%) & \multicolumn{2}{|c|}{12,57} & \multicolumn{2}{|c|}{0,61} \\
\hline
\end{tabular}

$\mathrm{O}$ alimento era ofertado diariamente às 08:00 e as 17:00 horas, dividido igualmente nos dois fornecimentos. $\mathrm{O}$ volumoso era colocado primeiramente no comedouro e o concentrado sobre ele. As quantidades ofertadas eram pesadas diariamente e ajustadas de acordo com o consumo dos animais, para proporcionar sobras diárias entre 15 e 20\%. As composições químico-bromatológicas das dietas estão apresentadas a seguir (Tabela 2).

As amostragens dos alimentos fornecidos, sobras e fezes foram realizadas diariamente durante os cinco dias de coleta. As fezes eram recolhidas em bandejas plásticas e a urina ficava acondicionada em baldes plásticos, adaptados com uma tela separadora, evitando que as fezes e a urina se misturassem. Cada balde de coleta de urina recebeu $100 \mathrm{~mL}$ de ácido sulfúrico $\left(\mathrm{H}_{2} \mathrm{SO}_{4}\right)$ a 2,0 $\mathrm{N}$ como conservante. 
Tabela 2: Composição bromatológica e química das dietas oferecidas aos animais com diferentes níveis de inclusão de torta de dendê

\begin{tabular}{|c|c|c|c|c|}
\hline \multirow{2}{*}{ Componentes } & \multicolumn{4}{|c|}{ \% Torta de dendê } \\
\hline & $\mathbf{0}$ & 7,5 & 15 & 22,5 \\
\hline \multicolumn{5}{|c|}{ Composição bromatológica e química das dietas } \\
\hline Matéria seca (\%) & 62,78 & 62,06 & 60,1 & 62,12 \\
\hline Proteína Bruta (\%) & 15,34 & 14,91 & 13,22 & 13,07 \\
\hline Energia Bruta $(\mathrm{cal} / \mathrm{g})$ & 3893 & 3946 & 3970 & 4120 \\
\hline Nutrientes digestiveis totais (\%) & 54,54 & 54,90 & 55,66 & 56,51 \\
\hline Extrato etéreo (\%) & 0,75 & 0,93 & 1,24 & 1,58 \\
\hline Lignina (\%) & 2,18 & 2,88 & 3,57 & 4,27 \\
\hline Celulose (\%) & 25,91 & 28,18 & 31,09 & 33,65 \\
\hline Hemicelulose (\%) & 26,15 & 25,51 & 23,8 & 20,96 \\
\hline Matéria mineral (\%) & 2,78 & 3,17 & 3,2 & 3,85 \\
\hline Carboidratos totais $(\%)$ & 81,13 & 80,99 & 82,34 & 81,51 \\
\hline Carboidratos não fibrosos (\%) & 26,89 & 24,42 & 23,88 & 22,63 \\
\hline Fibra em detergente neutro (\%) & 54,24 & 56,57 & 58,46 & 58,88 \\
\hline Fibra em detergente ácido (\%) & 28,09 & 31,06 & 34,66 & 37,92 \\
\hline NIDA $(\%)^{1}$ & $0,86(5,61)$ & $1,22(8,18)$ & $1,59(12,01)$ & $1,95(14,92)$ \\
\hline NIDN $(\%)^{1}$ & $1,29(8,41)$ & $2,12(14,22)$ & $2,94(22,24)$ & $3,76(28,77)$ \\
\hline
\end{tabular}

NIDA=nitrogênio insolúvel em detergente ácido; NIDN=nitrogênio insolúvel em detergente neutro; ${ }^{1}=\mathrm{Os}_{\text {s }}$ valores entre parênteses correspondem ao valor expresso em \% do nitrogênio total.

Após homogeneização das fezes, sub-amostras de aproximadamente $15 \%$ do total de cada coleta foram retiradas e acondicionadas em sacos plásticos, identificados e mantidos em congelador a $-10{ }^{\circ} \mathrm{C}$. As coletas de amostras de urina eram realizadas pela manhã e à tarde, sendo retiradas alíquotas de aproximadamente $15 \%$ do total. Antes de serem armazenadas, foram filtradas com gaze, para a retirada de possíveis contaminações, com seu volume mensurado em proveta de vidro graduada.

O alimento recusado pelos animais (sobras) era coletado individualmente, antes de a refeição seguinte ser ofertada, sendo pesado e amostrado diariamente. A avaliação do consumo voluntário dos nutrientes foi determinada pela diferença entre a quantidade de nutrientes no material fornecido aos animais e a quantidade nas sobras nos comedouros. Nas amostras de alimento ofertado e recusado foram determinadas: matéria seca (MS); fibra em detergente neutro (FDN); fibra em detergente ácido (FDA); lignina (LIG); proteína bruta $(\mathrm{PB})$; nitrogênio insolúvel em detergente ácido (NIDA); nitrogênio insolúvel em detergente neutro (NIDN); extrato etéreo (EE), energia bruta (EB) e matéria mineral (MM). Nas amostras de fezes foram determinadas: matéria seca (MS); fibra em detergente neutro (FDN); fibra em detergente ácido (FDA); proteína bruta (PB) e energia bruta (EB). Nas amostras de urina foram feitas análises de MS, nitrogênio e EB.

As análises químico-bromatológicas foram efetuadas no Laboratório de Nutrição Animal da Universidade Federal de Uberlândia para determinação de MS, PB, EE, MM, EB, FDN, FDA, LIG, NIDA e NIDN ${ }^{(8,9)}$.

A determinação do perfil granulométrico das sobras e da silagem oferecida foi embasada na metodologia da separação de partículas por peneiras utilizando-se o equipamento Penn State Particle Size Separator, definindo-se a proporção de material com diâmetro superior a $19 \mathrm{~mm}$, entre 
19 e $8 \mathrm{~mm}$, entre 8 e $1,3 \mathrm{~mm}$ e menor que $1,3 \mathrm{~mm}$.

A porcentagem de hemicelulose foi obtida pela diferença entre FDN e FDA e a de celulose pela diferença entre FDA e LIG. Os valores dos nutrientes digestíveis totais (NDT) foram obtidos a partir da composição dos alimentos através das equações propostas por Sniffen et al. ${ }^{(10)}$. A porcentagem de carboidratos totais (CHOT) foi obtida pela equação de Sniffen et al. ${ }^{(10)}$ : CHOT = $100-(\% \mathrm{~PB}+\% \mathrm{EE}+\% \mathrm{MM})$; e a porcentagem dos carboidratos não fibrosos (CNF) foi obtida por: $\mathrm{CNF}=\mathrm{CHOT}-\mathrm{FDN}$.

Os coeficientes de digestibilidade aparente (DA) da MS, PB, FDN, HEMI, CEL e EB foram obtidos pela fórmula descrita por Martins et al. ${ }^{(11)}$ : DA $=[(\mathrm{kgcons} * \%$ cons $)-(\mathrm{kgfz} * \% \mathrm{fz}) /$ $(\mathrm{kgcons} * \%$ cons $)]^{*} 100$, em que: kgcons $=$ quantidade de alimento consumido; $\%$ cons $=$ teor do nutriente no alimento consumido; $\mathrm{kgfz}=$ quantidade de fezes coletadas; $\% \mathrm{fz}=$ teor do nutriente nas fezes.

Os valores de energia digestível (ED) foram obtidos pela diferença ente a energia bruta ingerida $(\mathrm{EBI})$ e a energia bruta fecal ( $\left.\mathrm{EB}_{\text {fecal }}\right)$. A energia metabolizável $(\mathrm{EM})$ foi obtida pela multiplicação da ED por $0,82^{(10)}$. Para cálculo dos coeficientes de metabolizabilidade foi feita a razão entre consumo de EM e consumo de EB.

O balanço aparente de nitrogênio foi calculado, de acordo com Decandia et al. ${ }^{(12)}$, pelas seguintes equações, e expresso em g/dia: BN ou $\mathrm{N}_{\text {retido }}=\mathrm{N}_{\text {ingerido }}-\left(\mathrm{N}_{\text {fezes }}+\mathrm{N}_{\text {urina }}\right) ; \mathrm{N}_{\text {absorvido }}=\mathrm{N}_{\text {ingerido }}-\mathrm{N}_{\text {fezes }}$; e $\mathrm{N}_{\text {ingerido }}=\mathrm{N}_{\text {ofertado }}-\mathrm{N}_{\text {sobras }}$.

Foi feito estudo de regressão visando-se determinar o nível ótimo de inclusão da torta de dendê, considerando 5\% de significância para equações e coeficientes de regressão, utilizando-se o programa SAS (SAS Institute. Statistical Analysis System: user's guide: statistic [CD-ROM]. Release 6.03. Cary: SAS Institute Inc., 1998.).

\section{Resultados e discussão}

A silagem fornecida aos animais apresentou teores de FDN e de FDA 20,33\% e 13,70\% maiores quando comparada à torta de dendê (Tabela 1), respectivamente. O teor de FDN e de FDA encontrado nas sobras do grupo controle e dos grupos que receberam a torta de dendê (Tabela 3), nos diferentes níveis de inclusão, permite inferir que em todos os tratamentos, os animais selecionaram o alimento fornecido, procurando ingerir mais o alimento concentrado do que o alimento volumoso.

A quantidade de FDN das sobras foi significativamente diferente entre o grupo com $0 \%$ de inclusão e os demais grupos $(\mathrm{P}<0,01)$; contudo, a proporção de concentrado entre tratamentos variou de 40 a $52 \%$ (Tabela 1), o que, consequentemente, reduziu a proporção de volumoso oferecida aos animais, com o aumento dos níveis de inclusão da torta de dendê. Por meio da equação de regressão, é possível perceber que, a partir dos níveis de inclusão de 15 e $16 \%$ da torta de dendê, as sobras de FDN tornaram-se maiores como consequência provável da rejeição do próprio concentrado por parte dos animais.

Os teores de CNF das dietas oferecidas se apresentaram percentualmente menores com o aumento dos níveis de inclusão da torta de dendê (Tabela 2). Provavelmente, a silagem contribuiu com maiores teores de CNF às dietas do que a torta de dendê, já que, com o aumento dos níveis de inclusão da torta, a proporção de volumoso diminuiu e, consequentemente, reduziu a de CNF. Os 
teores de CNF das sobras apresentaram comportamento quadrático $(\mathrm{P}<0,01)$ com valores máximos no nível com $13 \%$ de inclusão da torta de dendê, o que sugere que, a partir deste nível, a seleção por parte dos animais procurando ingerir porções menos fibrosas do alimento foi mais intensa.

A ausência de diferenças significativas nos teores de lignina (LIG) das sobras $(\mathrm{P}>0,05)$ também pode justificar a preferência dos animais ao concentrado em detrimento do volumoso. Já que os teores de LIG na dieta ofertada aumentaram de 32,1\% a 95,8\% com a inclusão da torta de dendê (Tabela 2).

Tabela 3: Valores médios dos nutrientes encontrados nas sobras do alimento oferecido aos animais nas diferentes dietas experimentais

\begin{tabular}{|c|c|c|c|c|c|}
\hline \multirow{2}{*}{ Item } & \multicolumn{4}{|c|}{ \% Torta de dendê } & \multirow[b]{2}{*}{$\mathbf{P}$} \\
\hline & $\mathbf{0 \%}$ & $7,5 \%$ & $15 \%$ & $22,5 \%$ & \\
\hline Matéria seca (\%) & 59,71 & 56,48 & 63,69 & 60,13 & NS \\
\hline Proteína bruta (\%) & 7,44 & 7,81 & 7,73 & 9,02 & NS \\
\hline Energia Bruta (cal/g) & 3898 & 3940 & 3919 & 3965 & NS \\
\hline Extrato etéreo (\%) & 0,73 & 0,96 & 0,83 & 1,02 & NS \\
\hline Matéria mineral (\%) & 4,35 & 5,87 & 5,65 & 5,61 & NS \\
\hline Fibra em detergente neutro (\%) & 76,11 & 71,65 & 71,13 & 71,51 & $* *$ \\
\hline Fibra em detergente ácido (\%) & 43,32 & 43,34 & 42,17 & 43,08 & NS \\
\hline Carboidratos não fibrosos (\%) & 11,37 & 13,71 & 14,66 & 12,84 & $* *$ \\
\hline Carboidratos totais (\%) & 87,48 & 85,36 & 85,79 & 84,35 & NS \\
\hline Lignina (\%) & 2,10 & 2,56 & 2,01 & 2,53 & NS \\
\hline \multicolumn{6}{|c|}{${ }^{*}=$ significativo a $5 \%$ de probabilidade; **=significativo a $1 \%$ de probabilidade; NS=não significativo; } \\
\hline \multicolumn{6}{|c|}{$\begin{array}{l}C V=\text { coeficiente de variação; } R^{2}=\text { coeficiente de determinação da equação de regressão; FDN=fibra em detergente } \\
\text { neutro; } C N F=\text { carboidratos não fibrosos; } \\
\text { FDN: } \hat{Y}=75,97379-0,68862 X+0,02213 X^{2}\left(C V=2,35 ; R^{2}=0,63\right) \text {. } \\
C N F: \hat{Y}=11,31047+0,48118 X-0,01822 X^{2}\left(C V=9,52 ; R^{2}=0,53\right) .\end{array}$} \\
\hline
\end{tabular}

A preferência dos animais ao concentrado em detrimento do volumoso, provavelmente, decorreu das condições experimentais deste estudo em que a silagem de Brachiaria brizantha cv. Marandu possuía partículas grandes que permitiam a seleção (Tabela 4) e pelo fato de que o capim se encontrava em estágio avançado de maturação no momento do corte para ensilagem, fato que também comprometeu a qualidade da mesma. Neste estágio, as plantas forrageiras diminuem a densidade e a proporção de folhas e aumentam a proporção de caule ${ }^{(13)}$, havendo um espessamento de parede celular da planta, o que resulta no incremento da concentração da FDN em detrimento do conteúdo celular ${ }^{(14)}$, desfavorecendo o consumo e a digestibilidade.

De acordo com Maggioni et al. ${ }^{(15)}$, algumas forragens só são consumidas por ruminantes quando são a única escolha, mas podem ser rejeitadas se existir o oferecimento de algum alimento alternativo. Dessa forma, em situações em que é permitida a rejeição de grande quantidade de alimento, como o ocorrido neste estudo, os animais podem escolher a parte de maior digestibilidade da dieta.

$\mathrm{O}$ consumo de matéria seca (CMS) pelos animais, tanto em g/dia, \%PV, quanto em relação ao $\mathrm{PV}^{0,75}$, apresentou comportamento linear decrescente $(\mathrm{P}<0,01)$ com a inclusão da torta de dendê nas dietas (Tabela 5). Essa redução no CMS pode ter sido desencadeada por diversos fatores como o consumo de lignina pelos animais, os teores de fibra das dietas, o consumo de EE e pela 
disponibilidade do $\mathrm{N}$ presente neste coproduto, mas provavelmente está mais relacionado aos primeiros citados (consumo de LIG e teores de fibra das dietas). Uma vez que os teores de LIG aumentaram nas dietas, à medida que a torta de dendê foi incluída, ao passo que os de hemicelulose (HEMI) diminuíram (Tabela 2). Isto explica os consumos de LIG e de HEMI pelos animais que apresentaram comportamento linear crescente $(\mathrm{P}<0,01)$ e decrescente $(\mathrm{P}<0,01)$, respectivamente (Tabela 5). Os teores de CEL aumentaram com a inclusão da torta de dendê nas dietas, porém, este aumento não foi suficiente para provocar diferenças significativas no consumo deste nutriente $(\mathrm{P}>0,05)$, provavelmente pelo comportamento seletivo dos animais.

Tabela 4: Proporção percentual (\%) do tamanho das partículas encontradas nas sobras e na silagem ofertada aos animais nos diferentes níveis de inclusão da torta de dendê

\begin{tabular}{|c|c|c|c|c|c|}
\hline \multirow{2}{*}{$\begin{array}{c}\text { Tamanho da } \\
\text { partícula }\end{array}$} & \multicolumn{4}{|c|}{$\%$ Torta de dendê } & \multirow{2}{*}{$\begin{array}{l}\text { Silagem } \\
\text { ofertada }\end{array}$} \\
\hline & $\mathbf{0}$ & 7,5 & 15 & 22,5 & \\
\hline$>19 \mathrm{~mm}$ & $57,51^{\mathrm{Aa}}$ & $64,69^{\mathrm{Aa}}$ & $57,05^{\mathrm{Aa}}$ & $53,13^{\mathrm{Aa}}$ & 71,43 \\
\hline $19-8 \mathrm{~mm}$ & $15,47^{\mathrm{Ba}}$ & $13,24^{\mathrm{Ba}}$ & $11,86^{\mathrm{Ba}}$ & $13,38^{\mathrm{Ba}}$ & 14,3 \\
\hline $8-1,3 \mathrm{~mm}$ & $12,21^{\mathrm{Ba}}$ & $9,96^{\mathrm{Ba}}$ & $14,23^{\mathrm{Ba}}$ & $14,18^{\mathrm{Ba}}$ & 7,14 \\
\hline$<1,3 \mathrm{~mm}$ & $14,82^{\mathrm{Ba}}$ & $12,12^{\mathrm{Ba}}$ & $16,86^{\mathrm{Ba}}$ & $19,31^{\mathrm{Ba}}$ & 7,1 \\
\hline Total & 100 & 100 & 100 & 100 & 100 \\
\hline
\end{tabular}

${ }^{a}$ Médias seguidas de letras minúsculas iguais na linha não diferem pelo teste Tukey $(\mathrm{P}>0,05) ;{ }^{\mathrm{A}}$ médias seguidas de letras maiúsculas distintas na coluna diferem pelo teste Tukey $(\mathrm{P}<0,01)$.

Os consumos de MS em todos os tratamentos encontraram-se acima dos níveis recomentados pelo $\mathrm{NRC}^{(7)}$ que são de $770 \mathrm{~g} / \mathrm{dia}$ ou $1,93 \%$ do PV para ovinos adultos em mantença (Tabela 5). Contudo, a inclusão da torta de dendê reduziu o CMS, em \%PV, no tratamento $22,5 \%$ em 0,53 pontos percentuais quando comparado ao grupo com $0 \%$ de inclusão, o que implica na redução de $195 \mathrm{~g}$ de MS/dia para animais com 36,8 kg de PV.

Tabela 5: Consumo de matéria seca (MS), pelos ovinos expresso em gramas por dia (g/dia), percentual do peso vivo (\%PV) e em relação ao peso metabólico ( $\left.\mathrm{PV}^{0,75}\right)$, consumo de lignina, hemicelulose (HEMI), celulose, fibra em detergente neutro (FDN) e fibra em detergente ácido (FDA), em g/dia, em função dos níveis de inclusão da torta de dendê

\begin{tabular}{|c|c|c|c|c|c|c|c|}
\hline \multirow{2}{*}{ Consumo } & \multicolumn{4}{|c|}{ \% Torta de dendê } & \multirow{2}{*}{ CV \% } & \multirow{2}{*}{ ER } & \multirow{2}{*}{$\mathbf{R}^{2}$} \\
\hline & $\mathbf{0}$ & 7,5 & 15 & 22,5 & & & \\
\hline MS (g/dia) & 966,30 & 933,07 & 794,08 & 783,71 & 22,95 & $\hat{Y}=972,13-8,82 X^{* *}$ & 0,12 \\
\hline MS (\%PV) & 2,62 & 2,54 & 2,23 & 2,09 & 16,84 & $\hat{\mathrm{Y}}=2,65-0,0235 \mathrm{X}^{* *}$ & 0,19 \\
\hline $\mathrm{MS}\left(\mathrm{PV}^{0,75}\right)$ & 64,44 & 62,27 & 54,35 & 51,60 & 17,14 & $\hat{\mathrm{Y}}=65,01-0,5826 \mathrm{X}^{* *}$ & 0,19 \\
\hline Lignina (g/dia) & 33,17 & 37,74 & 46,47 & 53,28 & 21,02 & $\hat{\mathrm{Y}}=32,32+0,9141 \mathrm{X}^{* *}$ & 0,43 \\
\hline HEMI (g/dia) & 166,32 & 166,36 & 102,47 & 71,64 & 39,59 & $\hat{\mathrm{Y}}=178,84-4,5782 \mathrm{X} * *$ & 0,35 \\
\hline Celulose (g/dia) & 192,73 & 203,09 & 168,69 & 165,64 & 35,76 & $\hat{\mathrm{Y}}=199,11^{\mathrm{NS}}$ & - \\
\hline FDN (g/dia) & 392,23 & 407,19 & 317,63 & 295,79 & 33,95 & $\hat{\mathrm{Y}}=410,28-4,9805 \mathrm{X}^{* *}$ & 0,11 \\
\hline FDA (g/dia) & 225,90 & 240,83 & 215,15 & 224,14 & 31,79 & $\hat{\mathrm{Y}}=231,44^{\mathrm{NS}}$ & - \\
\hline
\end{tabular}

$\mathrm{CV}=$ coeficiente de variação; $\mathrm{ER}=$ equação de regressão; $\mathrm{R}^{2}=$ coeficiente de determinação da equação de regressão; $\mathrm{X}=$ nivel da torta; ${ }^{* *}=$ significativo a $1 \%$ de probabilidade; NS=não significativo. 
A inclusão da torta de dendê elevou a porcentagem de FDN e de FDA nas dietas (Tabela 2); entretanto, à medida que se aumentou o nível do coproduto houve decréscimo no consumo de FDN $(\mathrm{P}<0,01)$ e para consumo de FDA não houve diferenças significativas $(\mathrm{P}>0,05)$. O consumo de FDN parece ter sido influenciado pelas proporções de cada componente da parede celular, pois quando se considerou a HEMI, que teve consumo linear decrescente, a ingestão de FDN seguiu o mesmo comportamento. Ao se avaliar FDA, esta apresentou o mesmo comportamento da CEL (sem diferenças significativas, $\mathrm{P}>0,05)$; no entanto, ao se analisar a LIG separadamente, seu consumo apresentou comportamento linear crescente $(\mathrm{P}<0,01)$ (Tabela 5). Provavelmente, este fato decorreu devido à seleção dos animais que, ao rejeitarem a silagem de $B$. decumbens cv. Marandu, acabaram ingerindo mais concentrado e, consequentemente, mais torta de dendê e, pelo tamanho da partícula ser de difícil seleção, isso fez com que os animais ingerissem maiores quantidades de LIG, com o aumento dos níveis de inclusão do coproduto.

De acordo com Van Soest ${ }^{(9)}$, o consumo é dependente do conteúdo de FDN das dietas e do teor de lignina, havendo forte correlação negativa entre a parede celular ou lignina e o CMS. Ainda segundo o autor, a fermentação e a passagem de FDN pelo retículo-rúmen são mais lentas que outros constituintes dietéticos quando comparadas aos componentes não fibrosos do alimento cuja a parede celular indigerível ocupa espaço no trato gastrintestinal provocando, assim, redução no consumo.

Os valores de FDN das dietas deste estudo variaram entre 54,24\% e 58,88\% (Tabela 2) e foi possível observar redução no CMS com esses teores. Segundo Macedo Junior et al. ${ }^{(16)}$, quando FDN está abaixo de 50 a $60 \%$, para animais adultos, o consumo pode ser limitado pela demanda de energia dos animais e não pelo efeito de enchimento do alimento. Porém, os valores mencionados acima são para a espécie bovina, que possui o rúmen proporcionalmente maior quando comparada à espécie ovina; contudo, é possível que, neste estudo, os níveis de FDN das dietas possam estar no limiar entre a interrupção física e a interrupção química do CMS pelos animais. Assim, os teores de fibra das dietas aliados ao crescente consumo de LIG pelos animais foram fatores limitantes para o CMS.

A torta de dendê utilizada neste estudo apresentou um teor de EE de 12,57\% na MS (Tabela 1) e o teor deste nutriente nas dietas foi diretamente proporcional à inclusão do coproduto nos tratamentos (Tabela 2). O nível 22,5\% de inclusão de torta de dendê na dieta foi o que proporcionou maior valor para consumo de EE em relação à matéria seca ingerida, que foi de 5,28\%.

Para a espécie bovina, teores superiores a 5,5\% de EE na MS da dieta podem comprometer o consumo $^{(17)}$; entretanto, para dietas de terminação com alto teor de concentrados, Hess et al. ${ }^{(18)}$ relataram que 9,4\% de adição de lipídios na matéria seca não afetou a digestibilidade de outros componentes da dieta. Resposta semelhante foi observada por Cunha et al. ${ }^{(19)}$, em estudo com ovinos, em que não encontraram redução no CMS mesmo nos níveis mais altos de inclusão de caroço de algodão integral na dieta total (40\%), que resultou em consumo de EE pelos animais de 8,9\% da MS ingerida/dia. Dessa forma, infere-se que o consumo linear crescente de EE pelos animais, no presente experimento (Tabela 6), provavelmente não foi fator decisivo no CMS pelos animais, já que o consumo crescente de LIG, já discutido, foi fator primário a limitar o CMS.

$\mathrm{O}$ consumo de $\mathrm{PB}$ apresentou comportamento linear decrescente $(\mathrm{P}<0,01)$ devido à redução no CMS (Tabela 6). Os animais que receberam o maior nível de inclusão da torta de dendê $(22,5 \%)$ ingeriram menor porcentagem de PB em relação à MS ingerida $(7,7 \%)$ e, em valores absolutos expressos em g de PB ingerida/dia, os consumos médios de PB para todos os tratamentos foram 
superiores a $59 \mathrm{~g} /$ dia (Tabela 6), quantidade recomendada pelo $\mathrm{NRC}^{(7)}$ para ovinos adultos em mantença. As dietas foram formuladas para serem isoprotéicas e os níveis de proteína das dietas consumidas neste estudo foram superiores a $7 \%$, porém boa parte do $\mathrm{N}$ da torta de dendê está ligada a componentes da fração fibrosa como NIDA e NIDN. O NIDN, apesar de possuir lenta degradação ruminal, é considerado digestível, já o nitrogênio na forma de NIDA parece ser resistente e praticamente indigestível, estando geralmente associado à lignina e outros compostos de difícil degradação ${ }^{(20,21)}$. Os níveis de NIDA representaram 5,61\%, 8,18\%, 12,01\% e 14,92\% do nitrogênio total das dietas contendo $0 \%, 7,5 \%, 15 \%$ e $22,5 \%$ de torta de dendê, respectivamente (Tabela 2). Isso fez com que a quantidade disponível de PB ingerida fosse de 95,93; 83,14; 63,5 e 51,06 g/dia para os tratamentos com $0 \%, 7,5 \%, 15 \%$ e $22,5 \%$, respectivamente. O nível de inclusão de $15 \%$ apresentou consumo deste nutriente bastante próximo das exigências e, para o nível de 22,5\%, o consumo de $\mathrm{PB}$ foi de $6,5 \%$ em relação à matéria seca ingerida e abaixo dos níveis recomendados pelo $\mathrm{NRC}^{(7)}$, que é de $59 \mathrm{~g} /$ dia para ovinos em mantença.

Dietas com teor de PB inferior a 7\% ou com baixa disponibilidade de nitrogênio podem reduzir a digestibilidade dos constituintes fibrosos da parede celular por deficiência de compostos nitrogenados para os microrganismos ruminais ${ }^{(9)}$. Esse foi observado no tratamento $22,5 \%$ de inclusão da torta de dendê que apresentou baixos valores para os coeficientes de digestibilidade da CEL e da HEMI (Tabela 7).

O consumo de CNF não apresentou diferenças significativas entre tratamentos (Tabela 6). Os teores de CNF nas dietas obtiveram variação máxima de 4,26\%, com menores valores apresentados pelo tratamento com 22,5\% de inclusão e os maiores pelo tratamento com $0 \%$ (Tabela 2). A ausência de diferenças significativas no consumo deste nutriente pode ser explicada pelo fato de que à medida que se aumentaram os níveis de inclusão do coproduto às dietas, os animais procuraram ingerir frações menos fibrosas do alimento.

Provavelmente influenciado pelo CMS dos animais, o consumo de NDT também apresentou comportamento linear decrescente $(\mathrm{P}<0,01)$ com o aumento dos níveis de inclusão da torta de dendê (Tabela 6). Mesmo havendo aumento no consumo de EE $(\mathrm{P}<0,01)$ e ausência de diferenças significativas $(\mathrm{P}>0,05)$ no consumo de $\mathrm{CNF}$ pelos animais, estes não foram suficientes para atribuir aumento no consumo de NDT que, parece, então, ter sido influenciado negativamente pelo aumento no consumo das frações fibrosas, especialmente a lignina. Os consumos médios de NDT para todos os tratamentos apresentaram valores superiores ao nível recomendado pelo $\mathrm{NRC}^{(7)}$, que é de $410 \mathrm{~g}$ de NDT/dia para ovinos em mantença.

Os teores de CHOT e de MM nas dietas obtiveram variações máximas de 1,35\% e 1,07\%, respectivamente, e o consumo desses nutrientes pelos animais apresentou comportamento linear decrescente $(\mathrm{P}<0,01)$ (Tabela 6). Provavelmente, este comportamento é explicado em decorrência do CMS que apresentou resposta linear decrescente $(\mathrm{P}<0,01)$ com o aumento dos níveis de inclusão da torta de dendê (Tabela 5).

Os coeficientes de digestibilidade da MS, FDN, HEMI, CEL, EB e PB apresentaram comportamento linear decrescente $(\mathrm{P}<0,05)$ com o aumento da torta de dendê nas dietas (Tabela 7). A inclusão da torta de dendê provocou redução no coeficiente de digestibilidade da MS (CDMS) em 13,69 pontos percentuais quando comparado ao grupo $0 \%$, que recebeu concentrado formulado à base de milho grão e farelo de soja, alimentos considerados de alta digestibilidade.

Neste estudo, a redução na digestibilidade da fibra (FDN, HEMI e CEL) (Tabela 7) com consequente redução nos CDA da MS e EB (Tabela 8) podem ser atribuídas ao consumo crescente 
de lignina (Tabela 5), ao baixo nível de nitrogênio e ao alto teor de extrato etéreo nas dietas com maior inclusão de torta de dendê.

Tabela 6: Consumo de extrato etéreo (EE), proteína bruta (PB), carboidratos não fibrosos (CNF), nutrientes digestíveis totais (NDT), carboidratos totais (CHOT) e matéria mineral (MM), em g/dia, pelos ovinos em função dos níveis de inclusão da torta de dendê

\begin{tabular}{|c|c|c|c|c|c|c|c|}
\hline \multirow{2}{*}{ Consumo (g/dia) } & \multicolumn{4}{|c|}{ \% Torta de dendê } & \multirow{2}{*}{ CV \% } & \multirow{2}{*}{ ER } & \multirow{2}{*}{$\mathbf{R}^{2}$} \\
\hline & $\mathbf{0}$ & 7,5 & 15 & 22,5 & & & \\
\hline $\mathrm{EE}$ & 23,20 & 28,72 & 34,50 & 41,38 & 19,14 & $\hat{\mathrm{Y}}=22,97+0,7928 \mathrm{X}^{* *}$ & 0,55 \\
\hline PB & 101,63 & 90,55 & 72,17 & 60,01 & 22,45 & $\hat{\mathrm{Y}}=102,22-1,8208 \mathrm{X}^{* *}$ & 0,4 \\
\hline $\mathrm{CNF}$ & 379,01 & 358,48 & 344,99 & 364,37 & 20,21 & $\hat{\mathrm{Y}}=371,61^{\mathrm{NS}}$ & - \\
\hline NDT & 558,20 & 550,70 & 485,78 & 476,90 & 21,84 & $\hat{\mathrm{Y}}=564,12-3,9724 \mathrm{X}^{* *}$ & 0,08 \\
\hline CHOT & 771,23 & 765,67 & 662,61 & 650,28 & 23,82 & $\hat{\mathrm{Y}}=781,90-5,9220 \mathrm{X}^{* *}$ & 0,08 \\
\hline $\mathrm{MM}$ & 53,00 & 41,07 & 33,01 & 30,94 & 29,02 & $\hat{\mathrm{Y}}=49,68-0,93850 \mathrm{X}^{* *}$ & 0,32 \\
\hline
\end{tabular}

$\mathrm{CV}=$ coeficiente de variação; $\mathrm{ER}=$ equação de regressão; $\mathrm{R}^{2}=$ coeficiente de determinação da equação de regressão; $\mathrm{X}=$ nível da torta; $* *=$ significativo a $1 \%$ de probabilidade; $\mathrm{NS}=$ não significativo.

A digestibilidade da parede celular é limitada primariamente pela LIG $^{(22)}$. A lignina prejudica a digestão dos polissacarídeos por ser tóxica aos microrganismos do rúmen, por impedir fisicamente a digestão e pela limitação da ação de enzimas hidrolíticas (causada pela natureza hidrofóbica dos polímeros de lignina $)^{(23)}$. De acordo Van Soest ${ }^{(9)}$, o efeito físico causado pela lignina ocorre pelo fato de que a LIG cobre CEL e HEMI protegendo-as do ataque dos microrganismos do rúmen, mecanismo conhecido como "Teoria da Incrustação"(24). Como já discutido, menor quantidade de compostos nitrogenados, provenientes da dieta, impedem a ação mais efetiva de microrganismos ruminais nos constituintes fibrosos da parede celular ${ }^{(9)}$. Os ácidos graxos insaturados são tóxicos para as bactérias gram-positivas ruminais, pois podem alterar a permeabilidade da membrana celular, o que reduz a capacidade da célula de regular o $\mathrm{pH}$ intracelular e, como consequência, a captação de nutrientes $^{(25)}$.

$\mathrm{O}$ CDA da PB apresentou comportamento linear decrescente $(\mathrm{P}<0,01)$ provavelmente pelo aumento dos teores de NIDA das dietas com a inclusão da torta de dendê (Tabela 2) que, segundo Van Soest $^{(9)}$, interferem na digestibilidade da PB por estarem comumente associados à LIG e a outros compostos de difícil degradação. O nível de inclusão de 22,5\% apresentou menor valor de CDA da PB (30,55\%). O consumo desse nutriente pelos animais neste tratamento $(60,01 \mathrm{~g} / \mathrm{dia})$ foi bastante próximo da exigência pra ovinos em mantença que, segundo o $\mathrm{NRC}^{(7)}$, é de $59 \mathrm{~g} / \mathrm{dia}$. Segundo Van Soest $^{(9)}$, porcentagens inadequadas de PB reduzem a digestão das fibras pelo comprometimento da função ruminal. Assim, no tratamento com 22,5\%, os baixos valores de digestibilidade obtidos também podem ter sido influenciados, além do consumo de LIG já discutido, pelo fato de a PB disponível aos animais estar abaixo das exigências.

A queda no CMS provocou redução no consumo de EB, que apresentou comportamento linear decrescente (Tabela 8). A EB fecal apresentou comportamento linear crescente com os níveis de inclusão da torta de dendê. Isso demonstra que nas fezes desses animais ficaram mais partes indigestíveis da dieta, as frações fibrosas, evidenciando a seleção pelos mesmos, dentro da oferta de alimentos $(\mathrm{P}<0,01)$, o que, consequentemente, reduziu a $\mathrm{ED}(\mathrm{P}<0,01)$.

A redução da digestibilidade da CEL e HEMI (Tabela 8), causadas pela LIG, através da "teoria da Incrustação"(24), pode ter sido a causa da perda desses compostos estruturais nas fezes, contribuindo 
para o aumento dos níveis de EB fecal. Esse fato pôde ser evidenciado pelos constituintes das sobras, já que mesmo que a inclusão da torta de dendê nas dietas tenha elevado os níveis de FDA em 9,83\% (Tabela 2), os teores de FDA nas sobras dos animais (Tabela 3) não apresentaram diferenças significativas $(\mathrm{P}>0,05)$, sugerindo que os animais ingeriram complexos lignocelulósicos, considerados de baixa digestibilidade, com o aumento de inclusão do coproduto.

Tabela 7: Coeficientes de digestibilidade aparente da matéria seca (CDMS), proteína bruta (CDPB), fibra em detergente neutro (CDFDN), hemicelulose (CDHEMI), celulose (CDCEL) e energia bruta (CDEB), em porcentagem (\%), em função dos níveis de inclusão da torta de dendê

\begin{tabular}{|c|c|c|c|c|c|c|c|}
\hline \multirow{2}{*}{ Coeficientes } & \multicolumn{4}{|c|}{$\%$ Torta de dendê } & \multirow{2}{*}{ CV \% } & \multirow{2}{*}{ ER } & \multirow{2}{*}{$\mathbf{R}^{2}$} \\
\hline & 0 & 7,5 & 15 & 22,5 & & & \\
\hline CDMS & 75,24 & 69,86 & 61,63 & 61,55 & 12,51 & $\hat{\mathrm{Y}}=71,00-0,3650 \mathrm{X}^{* *}$ & 0,12 \\
\hline CDPB & 58,73 & 47,89 & 43,98 & 30,55 & 40,63 & $\mathrm{Y}=58,33-1,1734 \mathrm{X}^{* *}$ & 0,2 \\
\hline CDFDN & 57,68 & 55,52 & 43,54 & 43,73 & 28,57 & $\hat{\mathrm{Y}}=58,67-0,7386 \mathrm{X}^{* *}$ & 0,12 \\
\hline CDHEMI & 66,31 & 74,43 & 54,23 & 42,86 & 25,78 & $\hat{\mathrm{Y}}=73,78-1,3126 \mathrm{X}^{* *}$ & 0,34 \\
\hline CDCEL & 57,39 & 47,15 & 44,68 & 34,92 & 38,60 & $\hat{\mathrm{Y}}=49,91-0,8189 \mathrm{X}^{* *}$ & 0,16 \\
\hline CDEB & 74,65 & 69,94 & 63,02 & 62,48 & 12,53 & $\hat{\mathrm{Y}}=70,49-0,2938 \mathrm{X}^{*}$ & 0,08 \\
\hline
\end{tabular}

$\mathrm{CV}=$ coeficiente de variação; $\mathrm{ER}=$ equação de regressão; $\mathrm{R}^{2}=$ coeficiente de determinação da equação de regressão; $\mathrm{X}=$ nível da torta; $* *=$ significativo a $1 \%$ de probabilidade; ${ }^{*}=$ significativo a $5 \%$ de probabilidade.

A EB urinária e a EM apresentaram comportamento linear decrescente $(\mathrm{P}<0,01)$. Segundo Menezes et al. ${ }^{(26)}$ a ureia é a principal forma de eliminação do $\mathrm{N}$ metabólico em ruminantes. Quando o aporte proteico dietético é baixo, ocorre decréscimo na concentração de amônia no rúmen, com uma consequente diminuição nos teores de ureia nos líquidos corpóreos ${ }^{(27)}$ e, consequentemente, de N. Portanto, o menor teor de $\mathrm{N}$ disponível na dieta dos animais, com o aumento da inclusão da torta de dendê nas dietas, provocou redução no $\mathrm{N}$ urinário (Tabela 9), o que, por sua vez, levou à redução da EB urinária encontrada e da EM por ser derivada da fórmula de EB (Tabela 8).

Tabela 8: Energia bruta ingerida (EBI), Energia bruta fecal $\left(\mathrm{EB}_{\mathrm{fecal}}\right)$, Energia digestível (ED), Energia bruta urinária (EB urinária), Energia metabolizável (EM) e Metabolizabilidade (EM/EB) em função dos níveis de inclusão da torta de dendê

\begin{tabular}{|c|c|c|c|c|c|c|c|}
\hline \multirow{2}{*}{$\begin{array}{l}\text { Item } \\
\text { (Kcal/ } \\
\text { dia) }\end{array}$} & \multicolumn{4}{|c|}{ \% Torta de dendê } & \multirow[b]{2}{*}{ CV \% } & \multirow[b]{2}{*}{ ER } & \multirow[b]{2}{*}{$\mathbf{R}^{2}$} \\
\hline & $\mathbf{0}$ & 7,5 & 15 & 22,5 & & & \\
\hline EBI & 3631,99 & 3643,58 & 3232,91 & 3227,22 & 23,01 & $\hat{\mathrm{Y}}=3681,42-21,59 \mathrm{X}^{*}$ & 0,05 \\
\hline $\mathrm{EB}_{\text {fecal }}$ & 872,42 & 1032,06 & 1111,09 & 1182,96 & 34,22 & $\hat{\mathrm{Y}}=897,02+13,61 \mathrm{X}^{* *}$ & 0,09 \\
\hline ED & 2759,57 & 2611,51 & 2121,82 & 2044,26 & 36,58 & $\hat{\mathrm{Y}}=2784,40-35,20 \mathrm{X}^{* *}$ & 0,10 \\
\hline $\mathrm{EB}_{\text {urinária }}$ & 72,97 & 59,90 & 41,52 & 36,44 & 36,08 & $\begin{array}{c}\hat{\mathrm{Y}}=72,12-1,7174 \mathrm{X}^{* *} \\
\hat{\mathrm{Y}}=2390,43-\end{array}$ & 0,35 \\
\hline $\begin{array}{l}\mathrm{EM} \\
\mathrm{EM} / \mathrm{EB}\end{array}$ & 2368,33 & 2241,58 & 1816,74 & 1749,09 & 38,8 & $30,4793 X^{* *}$ & 0,09 \\
\hline$(\%)$ & 63,91 & 60,27 & 53,58 & 53,71 & 20,75 & $\hat{Y}=0,6368-0,00523 X^{* *}$ & 0,12 \\
\hline
\end{tabular}

$\mathrm{CV}=$ coeficiente de variação; $\mathrm{ER}=$ equação de regressão; $\mathrm{R}^{2}=$ coeficiente de determinação da equação de regressão; $\mathrm{X}=$ nivel da torta; ${ }^{* *}=$ significativo a $1 \%$ de probabilidade; ${ }^{*}=$ significativo a $5 \%$ de probabilidade. 
A metabolizabilidade da energia apresentou comportamento linear decrescente $(\mathrm{P}<0,01)$ com o aumento dos níveis da torta de dendê nas dietas (Tabela 8). A inclusão da torta de dendê em 7,5; 15 e $22,5 \%$ provocou redução na metabolizabilidade em 3,64; 10,33 e 10,20 pontos percentuais quando comparado ao grupo com $0 \%$ de inclusão, respectivamente.

$\mathrm{O}$ consumo de $\mathrm{N}$ apresentou comportamento linear decrescente $(\mathrm{P}<0,01)$ com a inclusão da torta de dendê nas dietas (Tabela 9), sendo que, no tratamento com 22,5\% de inclusão, a ingestão de $\mathrm{N}$ foi $33,43 \%$ menor quando comparada ao grupo com $0 \%$ de inclusão.

Apesar dessa redução no consumo de $\mathrm{N}$, o $\mathrm{N}$ fecal não apresentou diferenças significativas entre tratamentos $(\mathrm{P}>0,05)$; contudo, quanto maior o nível da torta de dendê nas dietas, menor foi o $\mathrm{N}$ absorvido $(\mathrm{P}<0,01)$. Essa resposta pode ser atribuída à queda da digestibilidade de $\mathrm{PB}$ (Tabela 7) e ao aumento de teores de NIDA e NIDIN que indisponibiliza o $\mathrm{N}$ ao animal, fazendo-o sair nas fezes.

O N urinário apresentou comportamento linear decrescente $(\mathrm{P}<0,01)$. Segundo Van Soest ${ }^{(9)}$, a excreção de $\mathrm{N}$ na urina é menor quando a concentração de $\mathrm{PB}$ da dieta e a ingestão de $\mathrm{N}$ pelo animal são diminuídas.

O balanço de nitrogênio (BN) apresentou comportamento linear decrescente $(\mathrm{P}<0,01)$, com os níveis de inclusão da torta de dendê nas dietas (Tabela 9), e pode ser explicado pela redução no consumo de nitrogênio $(\mathrm{P}<0,01)$ e pela redução na disponibilidade do mesmo (devido ao aumento de NIDA e NIDN) com o aumento dos níveis da torta de dendê. Os valores do BN para todos os tratamentos foram positivos, o que permitiu inferir que a exigência de proteína dos animais foi alcançada, porém, por se tratar de um balanço de nitrogênio aparente, esses valores estão superestimados, já que nesta variável a produção endógena de $\mathrm{N}$ não está descontada.

Tabela 9: Nitrogênios Ingerido ( $\mathrm{N}$ ingerido), Fecal ( $\mathrm{N}$ fecal), Urinário ( $\mathrm{N}$ urinário), Absorvido ( $\mathrm{N}$ absorvido) e Balanço de Nitrogênio, em g/dia, e porcentagem de Nitrogênio fecal e urinário em relação ao Nitrogênio ingerido em função dos níveis de inclusão da torta de dendê

\begin{tabular}{|c|c|c|c|c|c|c|c|}
\hline \multirow{2}{*}{ Item } & \multicolumn{4}{|c|}{$\%$ Torta de dendê } & \multirow{2}{*}{ CV \% } & \multirow{2}{*}{ ER } & \multirow{2}{*}{$\mathbf{R}^{2}$} \\
\hline & $\mathbf{0}$ & 7,5 & 15 & 22,5 & & & \\
\hline $\mathrm{N}$ ingerido & 23,27 & 20,41 & 17,3 & 15,49 & 19,62 & $\hat{\mathrm{Y}}=23,11-0,3543 \mathrm{X}^{* *}$ & 0,38 \\
\hline $\mathrm{N}$ fecal & 6,11 & 6,93 & 6,24 & 6,53 & 34,22 & $\hat{\mathrm{Y}}=6,37^{\mathrm{NS}}$ & - \\
\hline $\mathrm{N}$ urinário & 6,87 & 5,3 & 3,94 & 3,15 & 37,08 & $\hat{\mathrm{Y}}=6,70-0,1674 \mathrm{X}^{* *}$ & 0,37 \\
\hline $\mathrm{N}$ absorvido & 17,16 & 13,48 & 11,07 & 8,96 & 35,59 & $\hat{\mathrm{Y}}=16,74-0,3626 \mathrm{X}^{* *}$ & 0,31 \\
\hline Balanço de Nitrogênio & 10,30 & 8,18 & 7,13 & 5,81 & 62,55 & $\hat{\mathrm{Y}}=10,04-0,1952 \mathrm{X}^{* *}$ & 0,10 \\
\hline
\end{tabular}

$\mathrm{CV}=$ coeficiente de variação; $\mathrm{ER}=$ equação de regressão; $\mathrm{R}^{2}=$ coeficiente de determinação da equação de regressão; $\mathrm{X}=$ nível da torta; $* *=$ significativo a $1 \%$ de probabilidade; NS=não significativo.

\section{Conclusões}

Níveis de inclusão da torta de dendê acima de 7,5\% na dieta de ovinos provocam redução no consumo de matéria seca e na digestibilidade de nutrientes com possível comprometimento do desempenho, especialmente, em animais de alta produção. 


\section{Referências}

1. Abdalla, A. L; Silva Filho, J. C; Godoi, A. R; Carmo, C. A; Eduardo. J. L. P. Utilização de subprodutos da indústria de biodiesel na alimentação de ruminantes. Revista Brasileira de Zootecnia, v.37, suplemento especial p.260-258, 2008

2. Costa A, Ferreira GDG, Araújo CV, Colodo, JCN, Moreira GR, Figueiredo MRP. Consumo e digestibilidade de dietas com níveis de torta de dendê para ovinos. Revista Brasileira de Saúde e Produção Animal. 2010; 11 (3): 783-792.

3. Maciel RP, Neiva JNM, Araujo VL, Cunha OFR, Paiva J, Restle J, Mendes QC, Lôbo RNB. Consumo, digestibilidade e desempenho de novilhas leiteiras alimentadas com dietas contendo torta de dendê. Revista Brasileira de Zootecnia. 2012, 41(3): 698-706.

4. Silva HGO, Pires AJV, Silva FF, Veloso CM, Carvalho GGP, Cezário AS, Santos CC. Digestibilidade aparente de dietas contendo farelo de cacau ou torta de dendê em cabras lactantes. Pesquisa Agropecuária Brasileira. 2005, 40(4): 405-411.

5. Cunha OFR, Neiva JNM, Maciel RP, Miotto FRC, Neiva ACGR, Restle J. Avaliação bioeconômica do uso da torta de dendê na alimentação de vacas leiteiras. Ciência Animal Brasileira. 2012, 13(3): 315-322.

6. Bringel LML, Neiva JNM, Araújo VL, Bomfim MAD, Restle J, Ferreira ACH, Lôbo RNB. Consumo, digestibilidade e balanço de nitrogênio em borregos alimentados com torta de dendê em substituição à silagem de capim-elefante. Revista Brasileira de Zootecnia. 2011, 40 (9): 1975-1983.

7. NRC. National Research Council. Nutrient requirements of small ruminants: sheep, goats, cervids and new world camelids. Washington: National Academy Press, 2007. 362p. English. www.nap.edu

8. Silva DJ, Queiroz AC. Análise de alimentos: métodos químicos e biológicos. 3.ed. Viçosa: Editora UFV, 2002. 235p. Portuguese.

9. Van Soest, PJ; Robertson, JB; Lewis, BA. Methods for dietary fiber, neutral detergent fiber andnonstarch polysaccharides in relation to animal nutrition. Journal of Dairy Science. v.74, n. 10, p.3583-3597, 1991.

10. Sniffen CJ, O'Connor JD, Van Soest PJ, Fox DG, Russel JB. A net carbohydrate and protein system for evaluating cattle diets. II. Carbohydrate and protein availability. Journal of Animal Science. 1992, 70 (11):3562-3577.

11. Martins, RGR.; Gonçalves, LC; Rodrigues, JAS; Pôssas, FP. Consumo e digestibilidade aparente da matéria seca, da proteína bruta e da energia de silagens de quatro genótipos de sorgo (Sorghum bicolor (L.) Moench) por ovinos. Arquivo Brasileiro de Medicina Veterinária e Zootecnia, v.55, p.341-345, 2003

12. Decandia M, Sitzia M, Cabiddu A, Kababya D, Molle G. The use of polyethylene glycol to reduce the anti-nutritional effects of tannins in goat fed woody species. Small Ruminant Research. 2000, 38 (2): 157164.

13. Bauer MO, Pacheco LPA, Chichorro JF, Vasconcelos LV, Pereira DFC. Produção e características estruturais de cinco forrageiras do gênero Brachiaria sob intensidades de cortes intermitentes. Ciência Animal Brasileira. 2011, 12 (1): 17-25.

14. Velásquez PAT, Berchielli TT, Reis RA, Rivera AR, Dian PHM, Teixeira IAMA. Composição química, fracionamento de carboidratos e proteínas e digestibilidade in vitro de forrageiras tropicais em diferentes idades de corte. Revista Brasileira de Zootecnia. 2010, 39 (6): 1206-1213.

15. Maggioni D, Marques JA, Rotta PP, Zawadzki F, Ito RH, Prado IN. Ingestão de alimentos. Semina: Ciências Agrárias. 2009, 30 (4):963-974.

Cienc. anim. bras. v.16,n.2, p. 179-192 abr./jun. 2015 
16. Macedo Junior GL, Pérez JRO, Vasconcelos e Almeida TR, Paula OJ, França PM, Assis RM. Influência de diferentes níveis de fdn dietético no consumo e digestibilidade aparente de ovelhas Santa Inês. Ciência e Agrotecnologia. 2006, 30 (3):547-553.

17. Allen MS. Effects of Diet on Short-Term Regulation of Feed Intake by Lactating Dairy Cattle. Journal of Dairy Science. 2000, 83(7): 1598-1624.

18. Hess BW, Moss GE, Rule DC. A decade of developments in the area of fat supplementation research with beef cattle and sheep. Journal of Animal Science. 2008, 86(14): E188- E204.

19. Cunha MGG, Carvalho FFR, Véras ASC, Batista AMV. Desempenho e digestibilidade aparente em ovinos confinados alimentados com dietas contendo níveis crescentes de caroço de algodão integral. Revista Brasileira de Zootecnia. 2008, 37(6):1103-1111.

20. Van Soest PJ, Mason VC. The influence of Maillard reaction upon the nutritive value of fibrous feeds. Animal Feed Science and Technology. 1991, 32(1-3):45-53.

21. Licitra G, Hernandez TM, Van Soest PJ. Standardization of procedures for nitrogen fractionation of ruminant feeds. Animal Feed Science and Technology. 1996, 57(4): 347-358.

22. Jung HG, Allen S. Characteristics of plant cell walls affecting intake and digestibility of forages by ruminants. Journal of Animal Science. 1995, 73 (9): 2774-2790.

23. Grabber JH. How do lignin composition, structure, and cross-linking affect degradability? A review of cell wall model studies. Crop Science. 2005, 45(3):820-831.

24. Van Soest PJ, McQueen RW. The chemistry and estimation of fibre. Proceedings of the Nutrition Society, 1973. 32 (3): 123-30.

25. NAGARAJA, T.G., AVEY, T.B., BARTLEY, E.E. 1982. Effect of lasalocid, monensin or thiopectin on lactic acidosis in cattle. Journal Animal Science, 54(3):649-658.

26. Menezes DR, Araújo GGL, Oliveira RL, Bagaldo AR, Silva TM, Santos AP. Balanço de nitrogênio e medida do teor de ureia no soro e na urina como monitores metabólicos de dietas contendo resíduo de uva de vitivinícolas para ovinos. Revista Brasileira de Saúde e Produção Animal. 2006, 7 (2); 169-175.

27. Mouro GF, Branco AF, Macedo FAF, Guimarães KC, Alcalde CR, Ferreira RA, Prohmann PEF. Substituição do milho pela farinha de mandioca de varredura em dietas de cabras em lactação: fermentação ruminal e concentração de ureia plasmática e no leite. Revista Brasileira de Zootecnia. 2002, 31 (4): 18401848. 